\title{
The Sympathetic Nervous System Contributes to Capsaicin-Evoked Mechanical Allodynia But Not Pinprick Hyperalgesia in Humans
}

\author{
Maywin Liu, ${ }^{1,3}$ Mitchell B. Max, ${ }^{1}$ Suzan Parada, ${ }^{2}$ Janet S. Rowan, ${ }^{2}$ and Gary J. Bennett ${ }^{1}$ \\ ${ }^{1}$ Neurobiology and Anesthesiology Branch, National Institute of Dental Research, and ${ }^{2}$ Clinical Center Nursing, \\ National Institutes of Health, Bethesda, Maryland 20892, and ${ }^{3}$ Department of Anesthesia, \\ Hospital of the University of Pennsylvania, Philadelphia, Pennsylvania 19104
}

The contribution of the sympathetic nervous system (SNS) to pain, mechanical allodynia (MA), and hyperalgesia in humans is controversial. A clearer understanding is crucial to guide therapeutic use of sympatholytic surgery, blocks, and drug treatments. In rats, capsaicin-evoked MA, and to some extent, pinprick hyperalgesia (PPH), can be blocked with $\alpha$-adrenoreceptor antagonists. In this study, we examined the contribution of the SNS to MA and PPH in normal human subjects by blocking $\alpha$-adrenoreceptors with intravenous phentolamine.

In a double-blinded, placebo-controlled, crossover study, subjects were given IV saline or phentolamine, $1 \mathrm{mg} / \mathrm{kg}$ over 20 min. Ten minutes after the start of the infusion, subjects received $100 \mu \mathrm{g}$ of intradermal capsaicin on the foot dorsum with the temperature of the injected site clamped at $36^{\circ} \mathrm{C}$. The temperature of the uninjected foot was used to monitor the degree of $\alpha$-adrenoreceptor blockade produced by phentolamine. Ongoing pain and MA and PPH areas were measured every $5 \mathrm{~min}$ for $60 \mathrm{~min}$.

A significantly greater increase in temperature on the uninjected foot was seen during the phentolamine infusion compared with the saline infusion, indicating $\alpha$-adrenergic blockade. Significantly less MA was observed with the phentolamine infusion 10-25 min after capsaicin injection than with the saline infusion. No significant differences in ongoing pain or PPH areas were seen between the two infusions at any time.

Our results suggest that capsaicin-evoked MA and PPH have different mechanisms, with the SNS having a role in MA but not in $\mathrm{PPH}$ or ongoing pain.

Key words: sympathetic nervous system; mechanical allodynia; mechanical hyperalgesia; capsaicin; phentolamine; pain
After some cases of nerve injury, pain phenomenon such as mechanical allodynia (MA), pain caused by normally innocuous stimuli) and pinprick hyperalgesia (PPH), greater pain than that normally induced by a painful stimulus) are often seen within and outside of the peripheral nerve territory of the injured area (Kelly, 1952; Dykes, 1984; Marchettini et al., 1992). The mechanisms of MA and PPH are not completely understood. One explanation is that these changes are caused by sensitization of secondary neurons of the CNS by intense peripheral nociceptor input, resulting in abnormal processing of painful and normally nonpainful stimuli. These neurons may require continued peripheral input to sustain these changes (Levine et al., 1985; LaMotte et al., 1991; Gracely et al., 1992). Animal data have indicated that the sympathetic nervous system (SNS) may contribute to this heightened peripheral input. In the normal, uninjured state, somatosensory primary afferent nociceptors do not appear to have sympathetic sensitivity. However, after injury, catecholamine sensitivity is often seen (Sato and Perl, 1991). Neuromas proximal to the site of injury develop ectopic discharges that increase with close intraarterial injection of catecholamines and with stimulation of the

Received June 10, 1996; revised Aug. 19, 1996; accepted Aug. 29, 1996.

We thank Elaine Robinovitz and Mary Wells for their help in conducting this study, Dr. Robert Coghill for help with the statistics, and Drs. Eli Eliav and Andrew Mannes for reviewing this manuscript.

Correspondence should be addressed to Dr. Mitchell B. Max, National Institutes of Health, 9000 Rockville Pike, Building 10, Room 3C-405, Bethesda, MD 20892.

Dr. Bennett's current address: Department of Neurology, Allegheny University of the Health Sciences, Philadelphia, PA 19102.

Copyright (C) 1996 Society for Neuroscience $0270-6474 / 96 / 167331-05 \$ 05.00 / 0$ sympathetic trunk (Wall and Gutnick, 1974; Korenman and Devor, 1981; Jänig, 1990).

In addition to nerve injury, pain produced by direct chemical activation of $\mathrm{C}$-fibers with capsaicin, the purified active ingredient of chili peppers, has been used as a model to study MA and PPH. Capsaicin has been shown to cause MA and PPH, with symptoms similar to those of nerve injury (Koltzenburg et al., 1992; Torebjörk et al., 1992). In both the nerve injury and capsaicin animal models, surgical and chemical sympathectomies and peripheral $\alpha$-adrenoreceptor antagonists have relieved MA (Kim and Chung, 1991; Kinnman and Levine, 1995).

Patients with MA, PPH, and trophic changes possibly suggestive of increased sympathetic tone often obtain relief after block of the sympathetic innervation to the affected area (Loh and Nathan, 1978; Raja et al., 1991). These patients are considered to have "sympathetically maintained pain" (SMP) (Roberts, 1986). Many of these patients also obtain relief with the nonspecific $\alpha$-adrenergic antagonist phentolamine and with regional sympatholytic treatment with guanethidine (Loh and Nathan, 1978; Blanchard et al., 1990; Raja et al., 1991). Phentolamine provides pain relief comparable with that seen with local anesthetic block of the sympathetic ganglion (Raja et al., 1991).

However, the importance of the SNS in sensory changes remains controversial. Some investigators claim that the sensory changes outside the territory of an injured nerve and the apparent relief with sympathetic block are attributable to psychological factors (Verdugo and Ochoa, 1994; Verdugo et al., 1994; Ochoa and Verdugo, 1995). An experimental model of SMP in normal volunteers would facilitate research into the sympathetic contri- 
bution to pain. In this study, we examined the possible role of the SNS, specifically the $\alpha$-adrenergic receptors, in the production of MA and PPH produced by capsaicin in normal human volunteers.

\section{MATERIALS AND METHODS}

Healthy volunteers were entered into this double-blinded, placebocontrolled, two-period crossover study. Because of the large variability in responses to capsaicin (Liu et al., 1995a; Park et al., 1995), all subjects were screened for their MA response to intradermal (ID) capsaicin before entry. Patients with MA responses $\geq 5 \mathrm{~cm}^{2}$ for at least $20 \mathrm{~min}$ advanced to the infusion portion of the study.

During all experimental sessions, subjects were placed in the seated position with their lower leg $135^{\circ}$ to their thigh. We preferred subjects to be in a sitting position, because we had used that position in a previous study of MA and PPH on the forearm and foot dorsum and found larger areas of MA and PPH on the foot dorsum (Liu et al., 1995b). In addition, we had observed lower cutaneous blood flow in the foot dorsum with the foot dependent compared with the foot placed at the same level as the legs, suggesting higher sympathetic tone in the dependent position (Liu M, Max MB, Robinovitz E, Gracely RH, Bennett GJ, unpublished observations). Subjects were asked to inform the investigators whether they had any sensations of the leg "falling asleep."

Because of the known temperature sensitivity of capsaicin-evoked sensory changes (Koltzenburg et al., 1992; Liu et al., 1995a), we stabilized the skin temperature of the injection site on the dorsum of the left foot at $36^{\circ} \mathrm{C}$ (range, $35.8-36.2^{\circ}$ ) using a feed-back-controlled heat lamp with the thermistor placed $1 \mathrm{~cm}$ from the injection site. Once the temperature was stable, $100 \mu \mathrm{g}(10 \mu \mathrm{l})$ of ID capsaicin (Fluka, Ronconocow, NY) in Tween 80 was injected into that foot. Temperature was monitored in the uninjected right foot to assess the effect of phentolamine on peripheral adrenoreceptors. Ongoing pain was measured at the time of injection using a $0-100,20 \mathrm{~cm}$ Visual Analog Scale (VAS). Ongoing pain, MA, and PPH were measured every $5 \mathrm{~min}$ after injection for a total of $60 \mathrm{~min}$. MA was measured using a \#2 flat artist's brush moving along eight radial spokes, beginning in an area with normal sensation $\sim 8 \mathrm{~cm}$ from the injection site and moving toward the site at a rate of $1 \mathrm{~cm} / \mathrm{sec}$, as described by Simone et al. (1989). To ensure beginning in an area of normal sensation, brush sensation of the injected foot $\sim 8 \mathrm{~cm}$ from the injection site was compared with the brush sensation of the uninjected foot $5 \mathrm{~min}$ after injection. Subjects were instructed to inform the investigator whether and when the sensation changed to "pain or discomfort." This spot was marked lightly with a marking pen. PPH was assessed using a $3.8 \mathrm{~cm}$ safety pin pressed lightly into the skin to cause a just barely visible indentation. Testing began $8 \mathrm{~cm}$ from the injection site, comparing the sensation with that of the uninjected foot to ensure that initial sites of stimulation had normal pinprick sensation. Subjects did not observe the sensory testing. Subjects were instructed to inform the investigators when the pinprick sensation changed into "something more painful or a different type of pain." If the subjects answered positively, he or she was asked to describe the sensation. This spot was then marked with a marking pen. The marks for the areas of MA and PPH were then transferred onto clear acetate sheets and connected to form polygons.

All sensory testing was done by three nurse investigators. Subjects who advanced to the phentolamine/placebo portion of the study had the same nurse for all three sessions. Nurse investigators were thoroughly trained in the brush and pin techniques before performing sensory testing on any subjects, and all were observed to perform the testing in a similar manner. Nurse investigators and the subjects were blinded as to the infusion condition. The physician monitoring the vital signs and temperature of the uninjected foot was not blinded but did not participate in the sensory testing. Pulse-oximetry, blood pressure, and ECG monitors were hidden from the view of the subjects and the nurses. Subjects were questioned for presyncopal symptoms (light-headedness, dizziness, nausea) 2 min before capsaicin injection and $30 \mathrm{sec}$ and $5 \mathrm{~min}$ after injection during each session.

Subjects were randomly assigned to either of two possible treatments orders: $1 \mathrm{mg} / \mathrm{kg}$ phentolamine (Ciba-Geigy, Summit, NJ) and saline or saline and phentolamine. A 20 gauge intravenous catheter was placed into a right antecubital vein, and a bolus of $1000 \mathrm{ml}$ of saline given over $30 \mathrm{~min}$ before the start of the phentolamine/placebo infusion as prophylaxis against hypotension that might result from phentolamine. Once the bolus was completed, the phentolamine/placebo solution $(100 \mathrm{ml})$ was infused over $20 \mathrm{~min}$ with a $2 \mathrm{ml} / \mathrm{kg} / \mathrm{hr}$ background infusion rate of saline. Infusion sessions were all conducted in the afternoon. Subjects were instructed to have both breakfast and lunch before the sessions. Sessions, including the capsaicin screen, were separated by a minimum of 1 week. The subject's surface anatomy (veins, nevi, scars, etc.) was traced onto acetate sheets and the point of injection marked on this sheet to ensure that areas used previously were not reinjected. To keep injection sites relatively uniform, sites were chosen so that they were 1-2 mm adjacent to veins on the mid-dorsum of the foot. Continuous EKG and pulseoximetry and intermittent blood pressure readings (every $10 \mathrm{~min}$ before the start of the drug infusion, every 2 min during the infusion and the initial $15 \mathrm{~min}$ after the completion of the infusion, and every $5 \mathrm{~min} 15 \mathrm{~min}$ after the end of the infusion) were obtained throughout each infusion session. The skin temperature of the left foot was stabilized, as described above. Temperature of the uninjected foot was measured continuously with a contact thermistor to assess presence of $\alpha$-adrenergic blockade. Ten minutes after the start of the phentolamine/placebo infusion, the subject was given $100 \mu \mathrm{g}$ of ID capsaicin to the foot dorsum.

MA and PPH areas were obtained by copying the acetate sheets onto standard photocopy paper, cutting out and weighing the obtained areas, and comparing these weights with the weight of a known area.

Statistical analysis. Because preliminary analysis revealed a nonGaussian distribution, MA and PPH areas for the two treatments at each time point were compared using the Wilcoxon signed rank test. Ongoing pain was analyzed using ANOVA with repeated measures. Peak temperature difference was analyzed using a $t$ test. All results are shown as mean \pm SEM.

\section{RESULTS}

Twenty-three subjects were prescreened with capsaicin to obtain the final sample size of 16 . Six subjects had inadequate MA areas during the screen, and one subject chose not to continue to the drug portion of the study. During the screening session, subjects with MA and PPH were asked to describe the sensation they had. MA was consistently described as a burning or tenderness and PPH was described as an initial increased sharpness followed by a burning sensation with removal of the pin. The study population consisted of 3 women and 13 men whose ages ranged from 22 to 39 years.

All subjects reported high pain VAS $10 \mathrm{sec}$ after capsaicin injection during both the phentolamine and placebo infusions, $86.5 \pm 1.2$ versus $85.5 \pm 1.2$, respectively, on a scale of 100 (NS). Significantly smaller MA areas were noted during the phentolamine session as compared with the placebo session at each time point from 10 to $25 \mathrm{~min}$ after capsaicin administration $(p<0.05)$ (Fig. 1). No significant difference in ongoing VAS pain scores were noted between the two sessions at any time point, although there was a nonsignificant tendency for lower pain with phentolamine ( $p>0.3$, ANOVA) (Fig. 2). There were no differences in $\mathrm{PPH}$ areas between the two sessions at any time point (Fig. 3). No differences in the descriptions of the PPH sensations were observed between the saline and phentolamine infusions.

Higher temperatures were seen in the uninjected foot from the end of the phentolamine infusion (10 min after capsaicin injection) to the end of the session (Fig. 4). A mean peak increase $( \pm \mathrm{SEM})$ in the temperature of the uninjected foot (measured before the start of the drug infusion to the peak temperature during the 60 min testing interval) of $2.9 \pm 0.6^{\circ} \mathrm{C}$ was seen in the phentolamine session versus $0.6 \pm 0.2^{\circ} \mathrm{C}$ in the placebo session $(p<0.001)$, indicating presence of $\alpha$-adrenergic blockade (Raja et al., 1991, 1996). On average, maximal temperature change was noted $15 \mathrm{~min}$ after the end of the infusion or $25 \mathrm{~min}$ after capsaicin injection (Fig. 4).

Vital signs remained stable through both infusions. Vasopressors were not required by any subject for treatment of hypotension during either infusion.

Unlike previous investigators (Raja et al., 1991), we did not use $\beta$-blockers to blunt any possible tachycardia. In three pilot pa- 


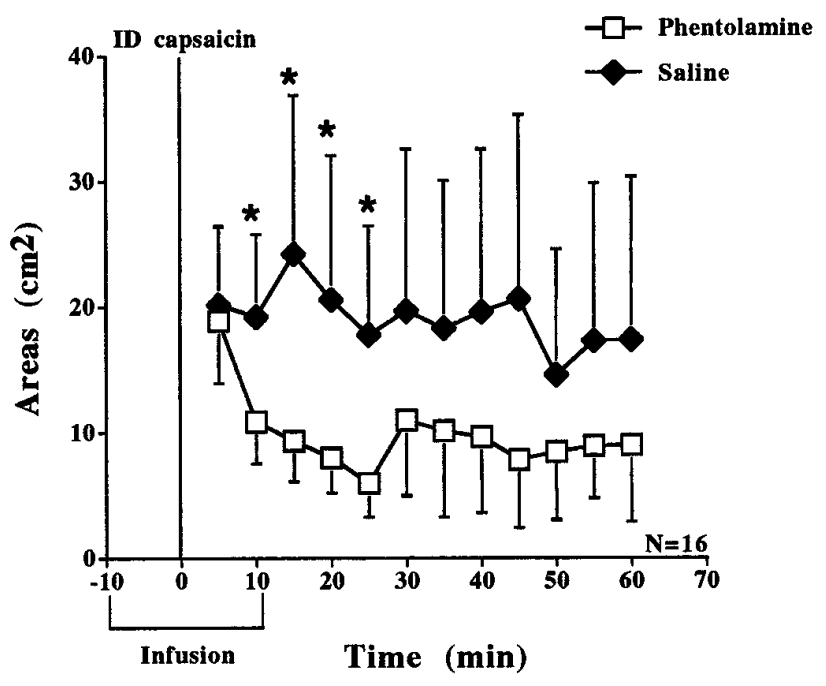

Figure 1. MA areas during the phentolamine (open squares) and saline (filled diamonds) infusions. Each point represents the mean areas of all 16 subjects at that time point \pm SEM. The vertical line at time " 0 " indicates the time of capsaicin injection. Phentolamine/saline infusions were started at -10 min and completed by +10 min. ${ }^{*} p<0.05$, areas of significantly decreased MA area during phentolamine infusion as compared with the saline infusion, Wilcoxon signed rank test.

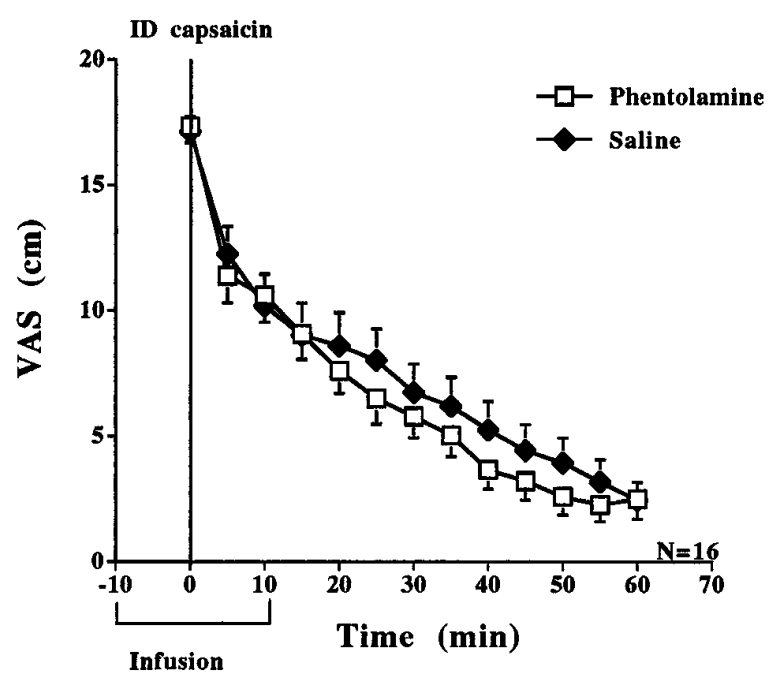

Figure 2. Ongoing pain measured on a $20 \mathrm{~cm}$ VAS during the phentolamine (open squares) and saline (filled diamonds) infusions. Each point represents the mean ratings for all 16 subjects at that time point \pm SEM.

tients, we had administered the recommended intravenous $1 \mathrm{mg}$ propranolol just before phentolamine infusion. Two of the three subjects developed near-syncopal symptoms consisting of complaints of nausea and light-headedness accompanied by bradycardia and hypotension $\sim 2-5$ min after capsaicin injection. We thought that this resulted from the blockade of hemodynamic compensatory mechanisms evoked by the combination of phentolamine and propranolol with the subject in the sitting position, causing unacceptable bradycardia and hypotension. Based on these reactions, propranolol was not used in our study and the prestudy saline bolus was increased from 500 to $1000 \mathrm{ml}$. No clinically significant incidents of bradycardia or hypotension were seen after the propranolol was discontinued. Although none of the subjects were informed of the symptoms of $\alpha$-adrenoreceptor block-

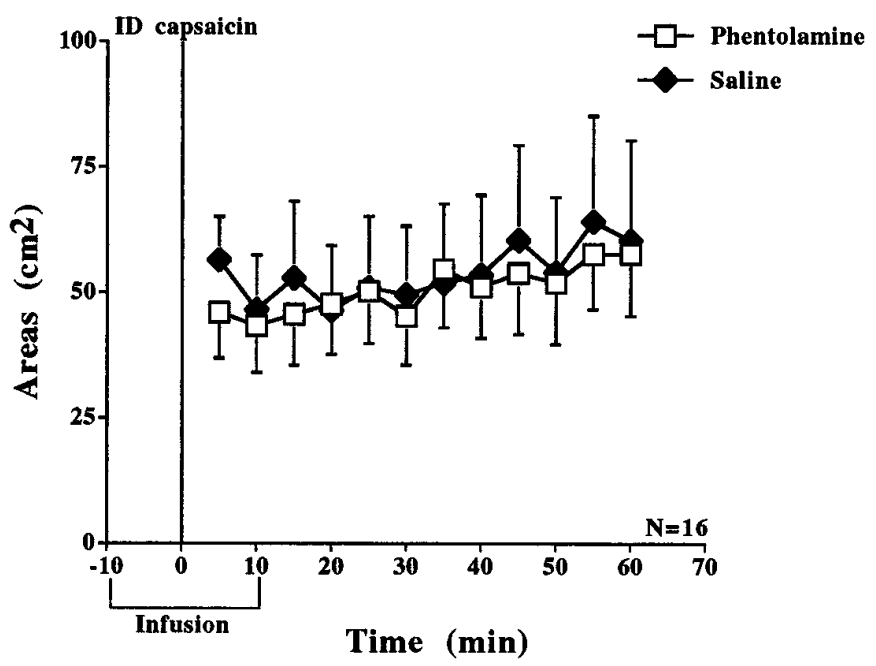

Figure 3. $\quad \mathrm{PPH}$ areas during the phentolamine (open squares) and saline (filled diamonds) infusions. Each point represents the mean areas of all 16 subjects at that time point \pm SEM.

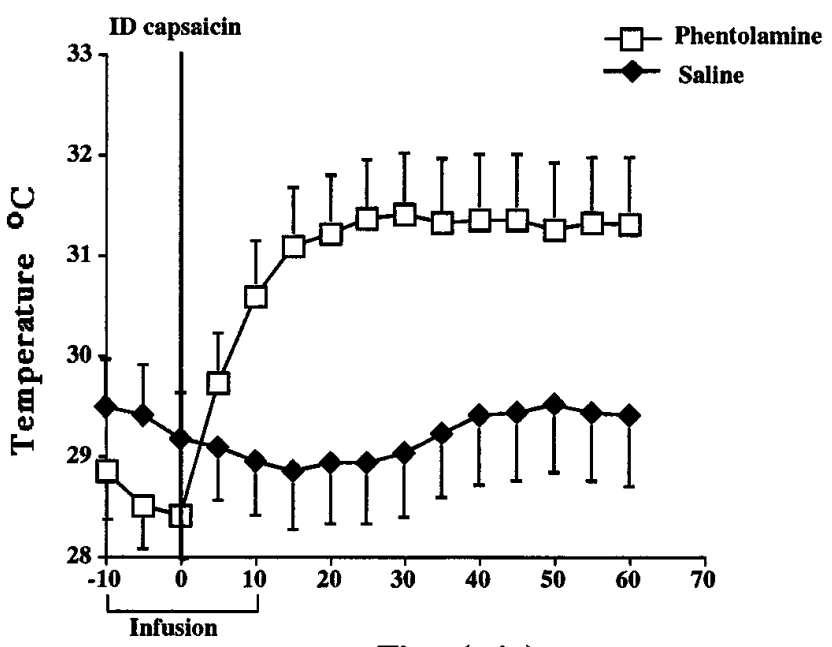

Time (min)

Figure 4. Skin temperatures. Mean temperature \pm SEM $\left({ }^{\circ} \mathrm{C}\right)$ during the course of the study for the phentolamine (open squares) and saline (filled diamonds) infusions as measured on the uninjected foot.

ade, 13 of the 16 subjects reported nasal stuffiness during the phentolamine infusion.

\section{DISCUSSION}

Our results extend to humans the findings obtained by Kinnman and Levine (1995) in rats. These investigators observed a complete or nearly complete prevention of capsaicin-evoked MA by phentolamine and by prazosin, a specific $\alpha-1$ adrenoreceptor antagonist. However, in our experiments, MA was not completely blocked by phentolamine. The discrepancy may be attributable to a species difference in the amount of sympathetic tone in the extremities, which is likely to be greater in humans because of the greater distance from the heart and the species difference in the thermoregulatory role of cutaneous blood flow (rats thermoregulate primarily by spreading saliva over their fur). In addition, the dose of phentolamine that we used $(1 \mathrm{mg} / \mathrm{kg}$ over $20 \mathrm{~min})$ results in only partial blockade $(\sim 88 \%)$ (Raja et al., 1994).

We observed a significant decrease in MA only during the 
period of 10-25 min after capsaicin injection. The lack of a significant effect at the 5 min test point, when $75 \%$ of the phentolamine dose had been delivered, may reflect an inadequate block. This is supported by the temperature data (Fig. 4), which showed little of the expected temperature increase at $5 \mathrm{~min}$ after capsaicin administration. The lack of a phentolamine effect after 25 min may have been at least partly attributable to the metabolism of phentolamine, the elimination half-life of which is $19 \mathrm{~min}$. The waning effect after $25 \mathrm{~min}$ was also probably attributable to the natural time course of capsaicin-evoked MA. As has been described previously, many subjects showed little MA 30 min after injection (Simone et al., 1989; LaMotte et al., 1991; Liu et al., 1995a; Park et al., 1995). The decrease in MA with phentolamine observed in the present study is not attributable to a temperature effect, because the site of capsaicin injection was kept at a fixed temperature of $36^{\circ} \mathrm{C}$, a temperature higher than the peak temperature reached in the uninjected foot during the phentolamine session.

Although we cannot rule out the possibility that phentolamineinduced nasal congestion unblinded the subjects and introduced self-report bias, we would have expected such a bias to produce similar effects on MA, PPH, and ongoing pain. Although the nurses doing the sensory testing could also potentially introduce bias after hearing subjects report this symptom, these clinicians had no investment in any particular outcome of the study, particularly the complex set of findings that we report.

The present data and the animal experiments of Kinnman and Levine (1995) suggest that capsaicin-evoked MA is reduced by blockade of sympathetic $\alpha$-adrenoreceptors. The mechanism of this effect is unknown.

A CNS mechanism is unlikely, because Kinnman and Levine (1995) showed that preganglionic sympathectomy has no effect on capsaicin-evoked MA. With the high dose of phentolamine used in the present experiments, we cannot exclude the possibility of a central effect of the drug. However, one would expect that central $\alpha$-adrenoreceptor block would increase pain via a reduction in the activity of the descending pain-inhibitory noradrenergic pathway that terminates in the spinal dorsal horn. Thus, it seems probable that the effects seen in the present experiments, like those of Kinnman and Levine (1995), are attributable to an effect in the periphery. There are two possible peripheral sites for an $\alpha$-adrenoreceptor effect on capsaicin-evoked MA: A $\beta$ lowthreshold mechanoreceptors (A $\beta$ LTMs) or C-fiber nociceptors.

An effect on $\mathrm{A} \beta$ LTMs is an unlikely explanation for our results. MA is evoked by A $\beta$ LTM stimulation (Torebjörk et al., 1992; Sang et al., 1996), and there is no reason to suspect that $\alpha$-adrenoreceptor blockade has any effect on stimulus-evoked activity in these afferents. Roberts (1986) has suggested that a tonic sympathetically maintained A $\beta$ LTM input sustains the hyperalgesic and allodynic sensations seen in nerve-injured patients. It is difficult to imagine how such a mechanism might be relevant to the present case, in which normal subjects exhibited phentolamine-blockable MA 10 min after capsaicin injection.

If there is an $\alpha$-adrenoreceptor effect that is necessary for or enhances the capsaicin-evoked C-nociceptor discharge, then $\alpha$-adrenoreceptor blockade would prevent or reduce the amount of central sensitization and thereby reduce the substrate for MA. However, there is no direct evidence that an $\alpha$-adrenoreceptor action on C-nociceptors modulates their activation by capsaicin. Capsaicin is an inflammatory stimulus and inflammation sensitizes C-nociceptors and "awakens" normally silent "sleepy" C-nociceptors (Handwerker et al., 1991;
Davis et al., 1993; Sato et al., 1993; Schmidt et al., 1995). If an $\alpha$-adrenoreceptor action is necessary for sensitization or for awakening sleepy nociceptors, then phentolamine block might have reduced the amount of $\mathrm{C}$-nociceptor activity during at least the latter stages of the postinjection interval. If this occurred, one would expect phentolamine to blunt the perceived intensity of capsaicin-evoked pain. Our results indicate a trend toward decreased pain with phentolamine, but this did not reach statistical significance $(p>0.3$; Fig. 2$)$.

Phentolamine had no effect on capsaicin-evoked PPH. Several lines of evidence indicate that capsaicin-evoked MA and PPH are at least partly independent phenomena. Capsaicin-evoked PPH is found in a distinctly larger area than MA, and it lasts a much longer time (Simone et al., 1989; LaMotte et al., 1991; Park et al., 1995). MA and PPH sensations are distinctly different qualitatively: MA is felt as a burning pain or a dysesthetic "raw" feeling like that of a sunburn; PPH is initially a distinctly localized, sharp, pricking pain with an electric shock-like component that evokes a flinch (M. Liu, unpublished observations). The afferent input that evokes PPH is not known, but it is likely to be $\mathrm{A} \delta$ - and/or C-fiber nociceptors. This is supported by a report of a patient with loss of $\mathrm{A} \beta$ function and preservation of $\mathrm{A} \delta$ and $\mathrm{C}$-fibers, showing that capsaicin produced ongoing pain and $\mathrm{PPH}$ but not MA (Treede and Cole, 1993). It may be of significance that trauma does not evoke $\alpha$-adrenergic sensitivity in A $\delta$ afferents (Bossut and Perl, 1995).

It has been argued that the relief of neuropathic pain in patients receiving sympathetic or $\alpha$-adrenoreceptor blockade is a placebo response and, further, that this and other evidence indicate that many patients diagnosed with SMP and related conditions suffer from a disorder that is primarily or exclusively psychogenic (Verdugo and Ochoa, 1994; Verdugo et al., 1994; Ochoa and Verdugo, 1995). The results reported here cannot be explained in this way, because neither the experimenters nor the subjects had any reason to believe that phentolamine would affect MA but not PPH or ongoing pain.

The human capsaicin model may be useful for elucidating the mechanisms by which sympathetic activity contributes to pain. MA and other abnormal pain sensations are exacerbated by $\alpha$-adrenoreceptor agonists (Drummond, 1995) and relieved by sympathetic nerve and $\alpha$-adrenoreceptor blockade in patients and animals with painful peripheral neuropathies (Bonica, 1990; Bennett and Roberts, 1996) and in animal models of inflammation (Levine et al., 1985, 1986; Hu and Zhou, 1989; Sato et al., 1993). The sensory changes obtained with capsaicin occur within minutes, and their mechanism may differ from those occurring in some chronic SMP syndromes in which new adrenoreceptor formation has been hypothesized. Although there may be differences in the sympathetic efferent and adrenoreceptor contribution to inflammatory, neuropathic, and capsaicin-evoked pain, our results suggest that animal and human capsaicin models may be used in tandem to work out the details of sympathetic interactions with each class of primary afferent and secondary neurons. The resulting insights into neural integration are likely to contribute to the understanding of many pain conditions.

\section{REFERENCES}

Bennett GJ, Roberts WJ (1996) Animal models and their contribution to our understanding of complex regional pain syndromes I and II. In: Reflex sympathetic dystrophy: a reappraisal. Progress in pain research and management, Vol 6, (Janïg W, Stanton-Hicks M, eds), pp 107-122. Seattle: IASP. 
Blanchard J, Ramamurthy S, Walsh N, Hoffman J, Schoenfeld L (1990) Intravenous regional sympatholysis: a double blind comparison of guanethidine, reserpine, and normal saline. J Pain Symptom Manage 6:357-361.

Bonica JJ (1990) Causalgia and other reflex sympathetic dystrophies. In: The management of pain, 2nd ed (Bonica JJ, ed), pp 220-243. Philadelphia: Lea \& Febiger.

Bossut DF, Perl ER (1995) Effects of nerve injury on sympathetic excitation of A $\delta$ mechanical nociceptors. J Neurophysiol 73:1721-1723.

Davis KD, Meyer RA, Campbell JN (1993) Chemosensitivity and sensitization of nociceptive afferents that innervate the hairy skin of monkey. J Neurophysiol 69:1071-1081.

Drummond PD (1995) Noradrenaline increases hyperalgesia to heat in skin sensitized by capsaicin. Pain 60:311-315.

Dykes RW (1984) Central consequences of peripheral nerve injuries. Ann Plast Surg 13:412-422.

Gracely RH, Lynch SA, Bennett GJ (1992) Painful neuropathy: altered central processing maintained dynamically by peripheral input. Pain 61:175-194.

Handwerker HO, Kilo S, Reeh RW (1991) Unresponsive afferent fibers in the sural nerve of the rat. J Physiol (Lond) 435:229-242.

$\mathrm{Hu}$ S, Zhu J (1989) Sympathetic facilitation of sustained discharges of polymodal nociceptors. Pain 38:85-89.

Jänig W (1990) Activation of afferent fibers ending in an old neuroma by sympathetic stimulation in the rat. Neurosci Lett 111:309-314.

Kelly M (1952) Spread of sensory and motor loss after nerve injury. Neurology 2:36-45.

Kim SH, Chung JM (1991) Sympathectomy alleviates mechanical allodynia in an experimental animal model for neuropathy in the rat. Neurosci Lett 134:131-134.

Kinnman E, Levine JD (1995) Involvement of the sympathetic postganglionic neuron in capsaicin-induced secondary hyperalgesia in the rat. Neuroscience 65:283-291.

Koltzenburg M, Lundberg LER, Torebjörk HE (1992) Dynamic and static components of mechanical hyperalgesia in human hairy skin. Pain 51:207-219.

Korenman EMD, Devor M (1981) Ectopic adrenergic sensitivity in damaged peripheral nerve axons in the rat. Exp Neurol 72:63-81.

LaMotte RH, Shain CN, Simone DA, Tsai E-FP (1991) Neurogenic hyperalgesia: psychophysical studies of underlying mechanisms. J Neurophysiol 66:190-211.

Levine JD, Dardick SJ, Basbaum AL, Scipio E (1985) Reflex neurogenic inflammation. I. Contribution of the peripheral nervous system to spatially remote inflammatory responses that follow injury. $\mathrm{J}$ Neurosci 5:1380-1386.

Levine JD, Taiwo YO, Collins SD, Tam JK (1986) Noradrenaline hyperalgesia is mediated through interaction with sympathetic postganglionic neurone terminals rather than activation of primary nociceptors. Nature 323:158-160.

Liu M, Max MB, Robinovitz E, Bennett GJ (1995a) Capsaicin-evoked allodynia: a study of three methods (abstract). Anesthesiology 83:A863.

Liu M, Max MB, Robinovitz E, Kingery EB, Gracely RH, Bennett GJ (1995b) Magnitude and variability of capsaicin-induced pain, allodynia, and pinprick hyperalgesia on foot dorsum compared to volar forearm, Am Pain Soc Meeting Abstracts A-38.

Loh L, Nathan PW (1978) Painful peripheral states and sympathetic blocks. J Neurol Neurosurg Psychiatry 41:664-671.

Marchettini P, Lacerenza M, Marangoni C, Pellagat G, Sotgiu ML, Smirne S (1992) Lidocaine test in neuralgia. Pain 48:377-382.

Ochoa JL, Verdugo RJ (1995) Reflex sympathetic dystrophy: a common clinical avenue for somatoform expression. Neurol Clin 13:351-363.

Park K, Max MB, Robinovitz E, Gracely RH, Bennett GJ (1995) Effects of intravenous ketamine, alfentanil, or placebo on pain, pinprick hyperalgesia, and allodynia produced by intradermal capsaicin in human subjects. Pain 63:163-172.

Raja SN, Treede R-D, Davis KD, Campbell JN (1991) Systemic alphaadrenergic blockade with phentolamine: a diagnostic test for sympathetically maintained pain. Anesthesiology 74:691-698.

Raja SN, Meleka SM, Turnquist JL, Campbell JN (1994) Monitoring adequacy of $\alpha$-adrenoreceptor blockade following systemic phentolamine administration. Am Pain Soc Meeting Abstracts 94A.

Raja SN, Turnquist JL, Meleka S, Campbell JN (1996) Monitoring adequacy of $\alpha$-adrenergic blockade following systemic phentolamine administration. Pain 64:197-204.

Roberts WJ (1986) A hypothesis on the physiological basis for causalgia and related pains. Pain 24:297-311.

Sang CN, Gracely RH, Max MB, Bennett GJ (1996) Capsaicin-evoked mechanical allodynia and hyperalgesia cross nerve territories: evidence for a central mechanism. Anesthesiology 85:491-496.

Sato J, Perl ER (1991) Adrenergic excitation of cutaneous pain receptors induced by peripheral nerve injury. Science 251:1608-1610.

Sato J, Suzuki S, Iseki T, Kumazawa T (1993) Adrenergic excitation of cutaneous nociceptors in chronically inflamed rats. Neurosci Lett 164:225-228.

Schmidt R, Schmelz M, Forster C, Ringkamp M, Torebjörk E, Handwerker H (1995) Novel classes of responsive and unresponsive C nociceptors in human skin. J Neurosci 15:333-341.

Simone DA, Baumann TK, LaMotte RH (1989) Dose-dependent pain and mechanical hyperalgesia after intradermal injection of capsaicin. Pain 38:99-107.

Torebjörk HE, Lundberg LER, LaMotte RH (1992) Central changes in processing of mechanoreceptive input in capsaicin-induced secondary hyperalgesia in humans. J Physiol (Lond) 448:765-780.

Treede R, Cole JD (1993) Dissociated secondary hyperalgesia in a subject with a large-fibre sensory neuropathy. Pain 53:169-174.

Verdugo RJ, Ochoa JL (1994) Sympathetically maintained pain. I. Phentolamine block questions the concept. Neurology 44:1003-1010.

Verdugo RJ, Campero M, Ochoa JL (1994) Phentolamine sympathetic block in painful neuropathies. II. Further questioning of the concept of "sympathetically maintained pain. Neurology 44:1010-1014.

Wall PD, Gutnick M (1974) Ongoing activity in peripheral nerves: the physiology and pharmacology of impulses originating from a neuroma. Exp Neurol 43:580-593. 